\title{
Metastable phases, phase transformation and properties of AlAs based on
} first-principle study

first-principle study

4 Chao Liu, Mengdong Ma, Xiaohong Yuan, Hao Sun, Pan Ying, Bo Xu, Zhisheng Zhao, and Julong 5 $\mathrm{He}^{\mathrm{a})}$

a) Author to whom correspondence should be addressed. E-mail address: hjl@ysu.edu.cn. 


\section{ABSTRACT}

2 By utilizing an evolutionary methodology on crystal structure search, we propose three new 3 metastable phases for aluminum arsenide (AlAs) as follows: (1) a $P 6_{4} 22$ symmetric structure 4 (hP6-AlAs), (2) a C222 symmetric structure (oC12-AlAs), and (3) a $I 3 d$ symmetric structure 5 (cI24-AlAs). By controlling the unloading pressure rate, oC12-, $h \mathrm{P} 6-$, and $c \mathrm{I} 24-\mathrm{AlAs}$ may be 6 acquired through quenching NiAs-AlAs. The elastic constants and phonon dispersion spectra are 7 calculated to certify the mechanical and dynamic stabilities of three newly discovered phases. On the basis of first-principle study, we explore phase transformations under pressure for several AlAs

9 polymorphs. The calculation of mechanical properties illustrates that $o \mathrm{C} 12-$, and $h \mathrm{P} 6-\mathrm{AlAs}$ possess

10 similar hardness levels, which are higher than that of $c$ I24-AlAs. Meanwhile, $o$ C12-, and $h \mathrm{P} 6-\mathrm{AlAs}$

11 hold similar shear anisotropic factors, which are smaller than that of cI24-AlAs. Electronic band

12 structure calculation reveals that at zero pressure, $o \mathrm{C} 12-$, and $h \mathrm{P} 6-\mathrm{AlAs}$ possess indirect band gaps of $13 \quad 0.468 \mathrm{eV}$ and $1.356 \mathrm{eV}$, respectively. $c \mathrm{I} 24-\mathrm{AlAs}$ is a direct semiconductor with a gap value $1.761 \mathrm{eV}$.

Keywords: aluminum arsenide; phase transformation; mechanical property; electronic property; 16 first-principle.

\section{Introduction}

At ambient conditions, aluminum arsenide (AlAs) exists stably with zinc blende structure (zb-AlAs), which is a common crystal form of III-V compounds. AlAs has excellent physical performances[1], including a high thermal expansion coefficient $5.20 \times 10^{-6} \cdot \mathrm{K}^{-1}$, thermal conductivity $0.9 \mathrm{~W} / \mathrm{cm} \cdot \mathrm{K}$, and, high melting points $2013 \mathrm{~K}$ and, and is an advantageous material for the manufacture of optoelectronic devices, such as light emitting diodes [2]. Given the almost identical

24 lattice constants, $\mathrm{AlAs}$ and gallium arsenide (GaAs) can form a superlattice $\mathrm{Al}_{\mathrm{X}} \mathrm{Ga}_{1-\mathrm{X}} \mathrm{As}$ with small 25 induced strain. With unique electrical and optical characteristics, $\mathrm{Al}_{\mathrm{X}} \mathrm{Ga}_{1-\mathrm{X}} \mathrm{As}$ holds important applications in optoelectronic devices, such as Bragg reflector superlattices, heterojunction bipolar transistors, solid-state lasers, high electron mobility transistors, and light emitting diodes $[2,3]$.

In 1983, Froyen and Cohen studied the structural properties of AlAs under pressure, and 
suggested that the high pressure structure transformed from zb-AlAs could be either rocksalt or NiAs (NiAs-AlAs) [4]. Several years later, Weinstein et al. observed a phase transformation within a high pressure experiment via diamond-anvil cell [5], and afterward, in a Raman study [6] at $~ 12.4$ GPa on uploading, however the structure was unknown. In the following years, experimental studies that explored the phase transition of AlAs have attracted increasing research attention. In 1994, Greene et al. first observed a first order phase transition from zb-AlAs to NiAs-AlAs at the equilibrium transformation pressure $7 \pm 5 \mathrm{GPa}$ through an energy-dispersive X-ray diffraction study on AlAs [7]. Subsequently, by X-ray diffraction and electrical resistivity measurements, zb-AlAs $\rightarrow$ NiAs-AlAs transformation was detected at 14.2 GPa on loading [8].

10 Theoretical research also achieved great progress in phases and phase transformation of AlAs 11 under high pressure. The calculated pressure of zb-AlAs $\rightarrow$ NiAs-AlAs phase transition ranges from 12 6.1 GPa to $9.15 \mathrm{GPa}$ [9-11]. Mujica et al. predicted that NiAs-AlAs becomes unstable and forms $13 \mathrm{CsCl}-\mathrm{AlAs}$ at $77.9 \mathrm{GPa}$ [11]. Other structures such as $\mathrm{cmcm}$, cinnabar, and sc16 of AlAs have been considered $[11,12]$. Research indicated $\mathrm{cmcm}$ structure AlAs (cmcm-AlAs) can stably exist within a certain pressure range, whereas the cinnabar and sc16 structures are not thermodynamically stable at any pressure and could occur only as metastable phases $[11,12]$. In 1992, wurtzite structure of AlAs has been proposed theoretically [6], and extensively investigated [13, 14].

In the present work, we proposed three new AlAs metastable phases. By analyzing elastic constants and phonon spectra, these AlAs polymorphs are found to be mechanically and dynamically stable, respectively. Then, we studied the phase transition behaviors under pressure, as well as the mechanical and electronic properties of these AlAs polymorphs.

\section{Computational methods}

The potential AlAs polymorphs' structures were explored by using the CALYPSO code without any experimental information [15-17], which has been widely used and has achieved substantial advances in structural prediction [18-22]. Once the structures were generated from the CALYPSO, geometric optimization, elastic constants and phonon frequency calculation, and physical property research were implemented in CASTEP code [23]. The exchange correlation potential generalized 
1 gradient approximation (GGA) of Perdew-Burke-Ernzerhof (PBE) was adopted [24, 25], and

2 atomic electronic configuration was described with the ultrasoft pseudopotential [26]. To ensure

3 calculation precision at $1 \mathrm{meV}$, the plane-wave pseudopotential method was employed under an

4 energy cutoff of $550 \mathrm{eV}$ and the k-points for Monkhorst-Pack grid was generated by a k-point 5 separation $\left(2 \pi \times 0.04 \AA^{-1}\right)[27]$.

6 GGA-PBE generally underestimates the energy gap by $30 \% \sim 40 \%$ relative to the experimental 7 value when calculating for band gap [28]. Hence, we adopted the Heyd-Scuseria-Ernzerhof hybrid 8 functional (HSE06) [29] on the primitive cells; such method can obtain an accurate prediction of 9 band gaps. Geometric optimization was carried out using the BFGS minimization algorithm [26],

10 during which iterations were continued until the following criteria were satisfied: (1) the force on 11 atom is less than $0.01 \mathrm{eV} / \AA$; (2) the atoms' displacement is below $5 \times 10^{-4} \AA$; (3) the energy change 12 does not go beyond $5 \times 10^{-6} \mathrm{eV} /$ atom; (4) the stress component does not exceed $0.02 \mathrm{GPa}$. The 13 efficient stress-strain method with the maximum strain amplitude 0.003 and 9 steps for each strain is 14 employed to calculate the elastic constants. To inspect dynamically stability of the obtained 15 structures, the phonon frequencies are calculated via the linear response method [30] as implemented 16 in CASTEP code [23].

\section{$18 \quad 3$. Results and discussion}

19 3.1. Optimisation of crystal structures
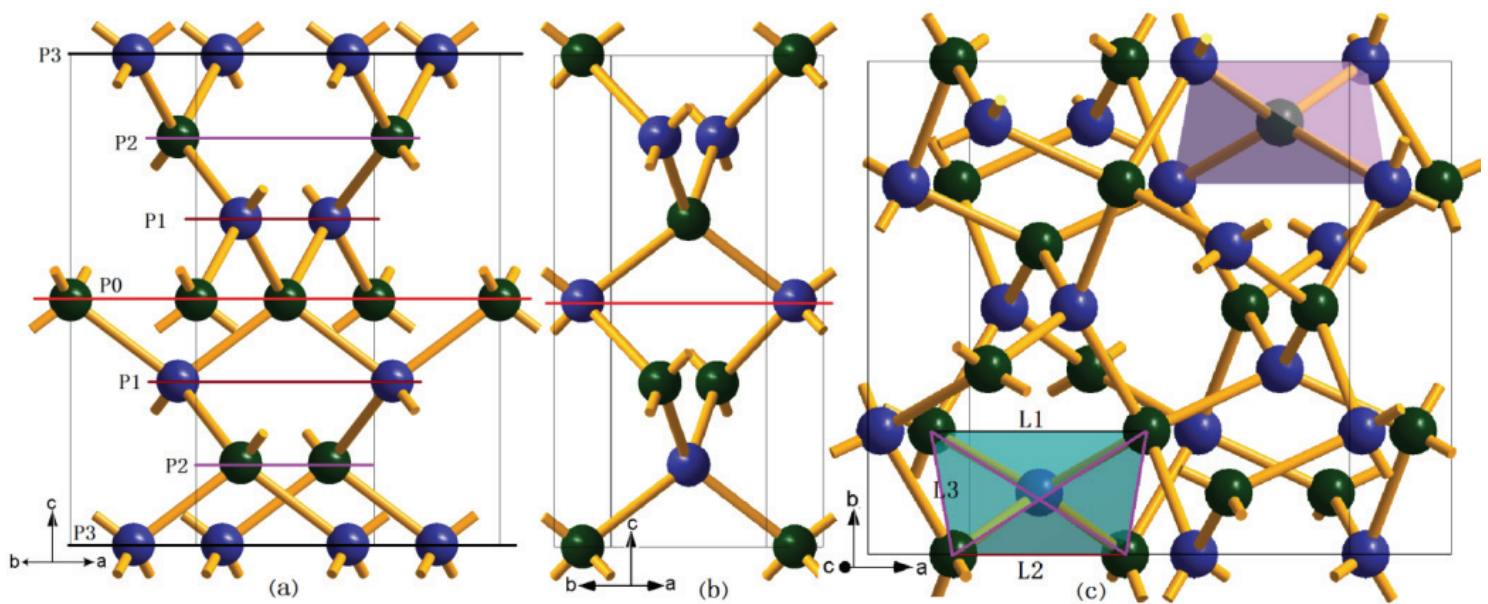

21 Fig. 1. Structure graphs for $o$ C12-AlAs (a), $h$ P6-AlAs (b), and $c$ I24-AlAs (c). The balls in dark green 
1 and indigo represent the $\mathrm{Al}$ and As atoms, respectively. The Al-As bonds are represented by gold 2 sticks.

A wide selection of candidate structures of AlAs was calculated. In addition to zb-AlAs,

5 NiAs-AlAs, cmcm-AlAs and the structures predecessors studied, three new structures attracted our

6 attention. The first new structure we proposed is a C-centered $(1 / 2,1 / 2,0)$ orthorhombic crystal system (space group C222) with 12 atoms per unit cell, denoted as $o$ C12-AlAs. The second one is a primitive centered hexagonal $\left(\mathrm{P}_{4} 22\right)$ structure with 6 atoms/unit cell, denoted as $h \mathrm{P} 6$-AlAs. The last one is a body centered cubic structure $(I 3 d)$ with 24 atoms per unit cell, denoted as $c$ I24-AlAs. All

10 atoms in $o \mathrm{C} 12-, h \mathrm{P} 6-$, and $c \mathrm{I} 24-\mathrm{AlAs}$ contain four coordination atoms, which combine to form Al-As

11 bonds, indicating that no Al-Al (As-As) bonds were present in the three newly discovered structures.

12 Information about $o \mathrm{C} 12-, h \mathrm{P} 6-$, and $c \mathrm{I} 24-\mathrm{AlAs}$, such as space group number, crystal system, lattice 13 parameter, density, and atomic Wyckoff positions, are listed in Table 1.

For $o \mathrm{C} 12-\mathrm{AlAs}$ (Fig. 1a), the atoms A12, As1, Al1 and As2 are distributed in the plane marked by $\mathrm{P} 0, \mathrm{P} 1, \mathrm{P} 2$ and $\mathrm{P} 3$, respectively. In $o \mathrm{C} 12-\mathrm{A} 1 \mathrm{As}$, three kinds of bonds with similar bond lengths are displayed as follows: the first one between P0 and P1 with length $2.512 \AA$; the second one between P1 and P2 with length $2.502 \AA$; and the last one with length $2.517 \AA$ are in P2 and P3. For $h$ P6-A1As (Fig. 1b), there is only one kind of Al-As bond with length $2.508 \AA$. It is a halved unit cell about the plane $c=0.5$, represented by a red line. Similar to $h$ P6-AlAs, only one kinds of bonds is shown present in $c$ I24-AlAs with bond length of $2.500 \AA$. The central atom $\mathrm{Al} / \mathrm{As}$ forms a tetrahedron $\left[\mathrm{AlAs}_{4}\right] /\left[\mathrm{AsAl}_{4}\right]$ with its 4 coordination $\mathrm{As} / \mathrm{Al}$ atoms, presented by a polyhedron in lavender/aqua, respectively. The tetrahedron $\left[\mathrm{AlAs}_{4}\right]$ is similar to $\left[\mathrm{AsAl}_{4}\right]$. The six edges that form the tetrahedron comprise one L1 (black), one L2 (wine) and four L3 (pink), marked by a polyhedron in aqua. L1 and L2 hold the same length of $4.562 \AA$; whereas the four L3 possess a uniform length of $3.817 \AA$. L1 is perpendicular to L2, however the two do not intersect as L1 and L2 are not in the same plane. One central atom connects with two coordination atoms and forms three atoms chain, the angle values of atoms chain are $99.594^{\circ}$ and $131.810^{\circ}$.

28 Referring to the optimized structures at zero pressure, cI24-A1As exhibits a slightly higher 
1 density than that of zb-AlAs $\left(3.604 \mathrm{~g} / \mathrm{cm}^{3}\right)$ by about $3.7 \%$. Meanwhile, both the $o \mathrm{C} 12-$ and $h \mathrm{P} 6-\mathrm{AlAs}$

2 possess higher densities than that of zb-AlAs by about $11.7 \%$ and $11.8 \%$, respectively.

4 Table 1

5 Space group (S.G.), crystal system (C.S.), lattice parameter (L.P.) ( $\AA$ ), density $\rho\left(\mathrm{g} / \mathrm{cm}^{3}\right)$, and atomic

6 Wyckoff positions (A.W.P) for three new AlAs polymorphs.

\begin{tabular}{lccccc}
\hline Structure & S.G. & C.S. & L.P. & $\rho$ & A.W.P. \\
\hline$o$ C12-A1As & $C 222(21)$ & orth & $a=6.975 ; b=3.977$ & 4.025 & Al1: $4 k(0.25,0.25,0.167) ;$ Al2: $2 d(0,0,0.5)$ \\
& & & $c=9.094$ & & As1: $4 k(0.25,0.25,0.666) ;$ As $2: 2 b(0.5,0,0)$ \\
& & & & & \\
$h$ P6-AlAs & $P 6422(181)$ & hex & $a=4.0260 ; c=8.973$ & 4.031 & Al: $3 c(0.5,0,1) ;$ As: $3 d(0.5,0,0.5)$ \\
$c$ I24-AlAs & $13 d(217)$ & cub & $a=8.161$ & 3.736 & Al: $12 a(0.375,0,0.25) ;$ As: $12 b(0.25,0.125,0.5)$ \\
\hline
\end{tabular}

7 orth: orthorhombic; hex: hexagonal; cub: cubic

9 3.2. Stability analysis

10 To verify the mechanical stability of $o \mathrm{C} 12-, h \mathrm{P} 6-$, and $c \mathrm{I} 24-\mathrm{Al} \mathrm{As}$, the elastic constants were 11 calculated and listed in Table 2.

12 For an orthorhombic crystal system, the mechanical stability under zero pressure can be 13 evaluated by [31]

$14 ; ;$

$15 ;$;

16

$17 \quad$ For cubic crystal system [31]:

$18 \quad ; ; ;$

19 For hexagonal crystal system [31]:

$20 \quad ; \%$

21 The calculated elastic constants at zero pressure satisfy the mechanical stability criteria, 22 suggesting that $o \mathrm{C} 12-, h \mathrm{P} 6-$, and $c \mathrm{I} 24-\mathrm{Al}$ As all possess mechanical stability. 


\section{Table 2}

2 Independent elastic constants $C_{i j}$ for three newly discovered AlAs polymorphs.

\begin{tabular}{llllllllll}
\hline Structure & $C_{11}$ & $C_{22}$ & $C_{33}$ & $C_{44}$ & $C_{55}$ & $C_{66}$ & $C_{12}$ & $C_{13}$ & $C_{23}$ \\
\hline$o$ C12-A1As & 126.9 & 121.2 & 152.8 & 46.6 & 38.2 & 42.8 & 38.8 & 45.0 & 51.6 \\
$h$ P6-AlAs & 125.7 & - & 146.8 & 44.3 & - & - & 38.1 & 50.8 & - \\
$c$ I24-AlAs & 104.0 & - & - & 41.9 & - & - & 49.1 & - & - \\
\hline
\end{tabular}

4 The appearance of soft phonon modes denotes the dynamical instability and will lead to

5 distortion of crystal. Phonon dispersions of $o \mathrm{C} 12-, h \mathrm{P} 6-$, and $c \mathrm{I} 24-\mathrm{AlAs}$ at zero pressure are

6 calculated and plotted in Fig. 2. There is no imaginary frequency in the entire Brillouin zone,

7 suggesting these new phases are all dynamically stable.

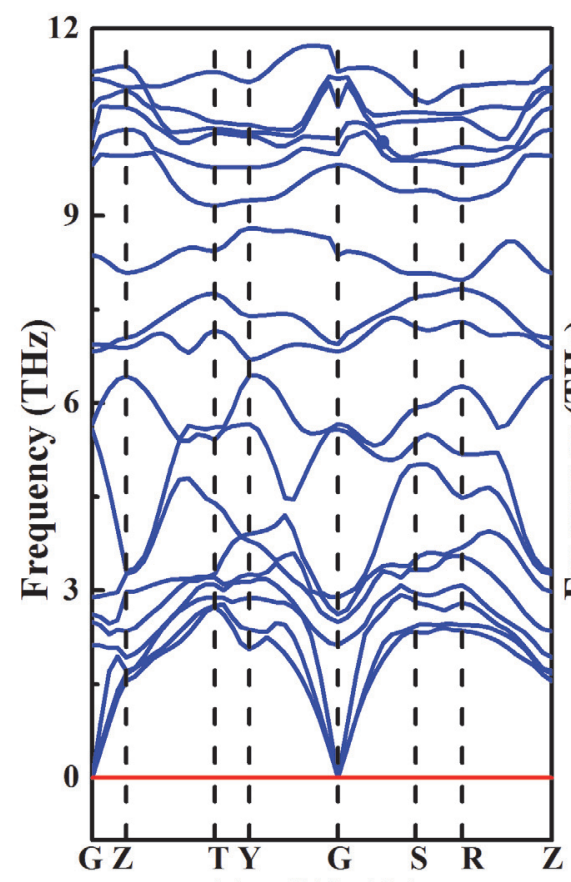

(a) oC12-AIAs

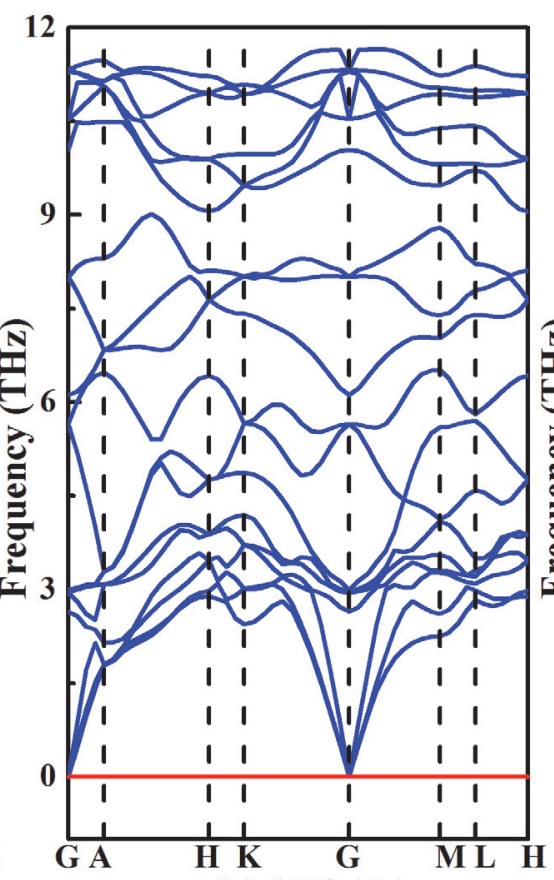

(b) hP6-AIAs

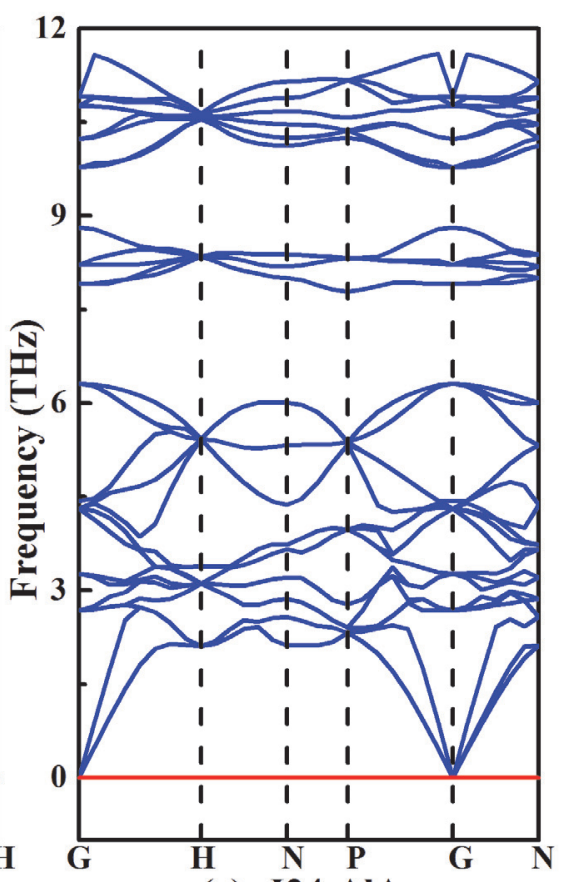

(c) $c$ I24-AIAs

Fig. 2. Phonon dispersion spectra for $o \mathrm{C} 12-\mathrm{AlAs}(\mathrm{a}), h \mathrm{P} 6-\mathrm{AlAs}(\mathrm{b})$, and $c \mathrm{I} 24-\mathrm{Al} A \mathrm{As}(\mathrm{c})$.

3.3. Phase transformation under pressure

13 Pressure, as a fundamental physical variable, notably affects the chemical and physical

14 properties of material. High-pressure technology is an interesting domain for material research. The 
1 enthalpy (per formula unit, f.u.) curves relative to zb-AlAs as a function of hydrostatic pressure up to

$260 \mathrm{GPa}$ for the AlAs polymorphs are presented in Fig. 3. It is clearly seen that zb-AlAs transforms to

3 NiAs-AlAs under the pressure $9.2 \mathrm{GPa}$, which not only agrees with experimental values $(7 \pm 5 \mathrm{GPa}$

4 [7] and $14.2 \mathrm{GPa}$ [8]) but also matches the theoretical values (5.34 [32], 6.1 [9], 6.99 [33], 7 [11],

59.15 [10] and 6.68 [34]). The calculated transformation pressure for NiAs-AlAs to cmcm-AlAs is

$634.4 \mathrm{GPa}$, which was consistent with the reported value $36 \mathrm{GPa}$ [12]. $o \mathrm{C} 12-, h \mathrm{P} 6-$, and $c \mathrm{I} 24-\mathrm{AlAs}$

7 are all pressure driven structures and hold lower energies than those of NiAs- and cmcm-AlAs at

8 ambient pressure. The decompression rate plays an important role during the metastable phase

9 formation, for example, the Si-XII, Si-III and Ge-III phases can be acquired by slowly releasing

10 pressure from Si-II and Ge-II, respectively [35, 36]. However, rapid depressurization causes the

11 formation of Ge-IV phase and Si-VIII/IX phases [37, 38]. hP6-AlAs will obtain its energy advantage

12 over that of zb-AlAs at 5.7 GPa during decompression. Meanwhile, $o$ C12-AlAs and $c$ I24-AlAs attain

13 its energy favorable than that of zb-AlAs at $2.3 \mathrm{GPa}$ and $2.5 \mathrm{GPa}$, respectively. Therefore, $o \mathrm{C} 12-$,

14 hP6-, and cI24-AlAs may be obtained by following steps: a) zb-AlAs loading pressure, b) forming

15 NiAs-AlAs, c) quenching NiAs-AlAs with controlled unloading rate.

16
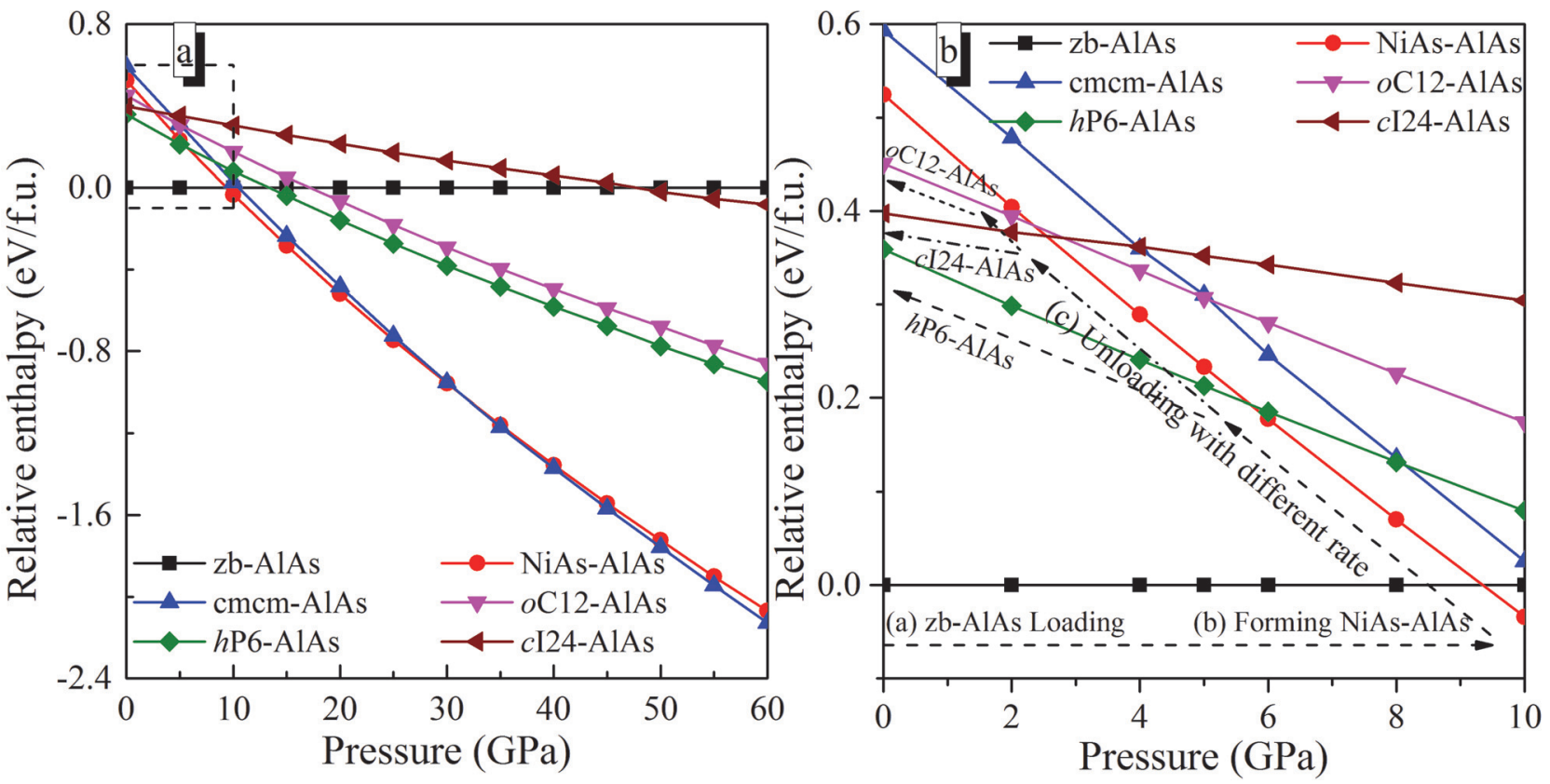

18 Fig. 3. Enthalpy curves (relative to zb-AlAs) as functions of pressure for various AlAs polymorphs.

$19 \mathrm{~b}$ is the zoomed in views of the selected region on the left panel. 
2 3.4. Mechanical properties

3 The pressure versus volume curves of $o \mathrm{C} 12-, h \mathrm{P} 6-$, and $c$ I24-AlAs are fitted to

4 Birch-Murnaghan equation of state (BM-EOS) [39].

$5 ;$

6 Herein, and $V$ represent the volume per formula unit at zero pressure and given pressure, 7 respectively; and represent the isothermal bulk modulus and its first pressure derivative, 8 respectively. The fitting results and a series value of pressure versus volume are presented in Fig. 4.

9 The obtained values of, and are listed in Table 3. Among the three new AlAs strcutures,

10 cI24-AlAs has the larges volume $\left(45.30 \AA^{3}\right)$ per formula unit at zero pressure and the largest volume 11 compressibility (32.70\%); oC12-AlAs and hP6-AlAs have the similar (42.06 $\AA^{3}$ and $41.97 \AA^{3}$, 12 respectively) and volume compressibility (31.82\% and $31.65 \%$, respectively).

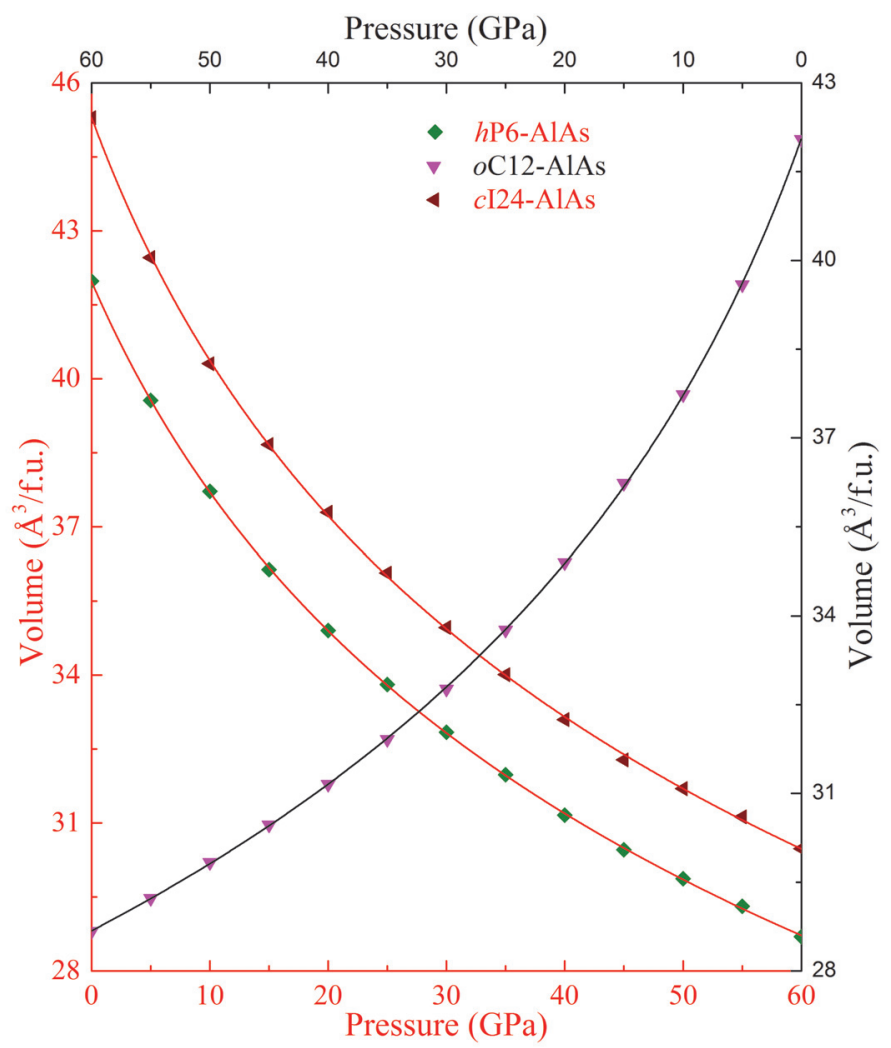

14 Fig. 4. Volumes of $o \mathrm{C} 12-, h \mathrm{P} 6-$, and $c \mathrm{I} 24-\mathrm{AlAs}$ as functions of pressure. The scatter symbol and 15 solid lines represent the calculated data and fitting results, respectively. 
1 Elasticity anisotropy is important for understanding the micro-cracks produced in ceramic

2 materials and significantly influences materials' engineering application [40]. As a widely used

3 criterion, the degree of anisotropy in the bonding between atoms in different planes can be measured

4 by the shear anisotropy. The shear anisotropic factors A1, A2 and A3 are described for shear planes

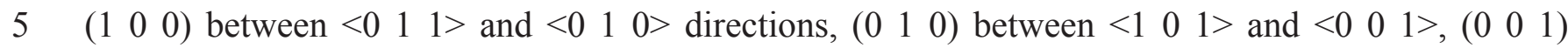

6 between $<1 \quad 10>$ and $<010>$, respectively [41].

$7 \quad ; ;$

$9 \quad$ For a cubic structure

11 For a hexagonal structure,

$12 ;$

13 For isotropic crystals, and are all equal to 1 . Any value deviation from 1 indicates the 14 degree of shear anisotropy. Our calculated shear anisotropic factors are given in Table 3. Among 15 three new AlAs polymorphs, $c$ I24-AlAs has the highest shear anisotropic factor, $h \mathrm{P} 6$-AlAs has the 16 isotropy for $\left(\begin{array}{lll}0 & 0 & 1\end{array}\right)$ shear plane, and slight anisotropy for $\left(\begin{array}{lll}1 & 0 & 0\end{array}\right)$ and $\left(\begin{array}{lll}0 & 1 & 0\end{array}\right)$ shear planes. For 17 oC12-AlP, all the shear planes $\left(\begin{array}{lll}1 & 0 & 0\end{array}\right),\left(\begin{array}{lll}0 & 1 & 0\end{array}\right)$ and $\left(\begin{array}{lll}0 & 0 & 1\end{array}\right)$ exhibit anisotropy.

18 The bulk modulus $(B)$ and shear modulus $(G)$ are calculated based on the elastic constants 19 [42-44], then Young's modulus $(E)$ and Poisson's ratio $(\mu)$ are obtained via Eq. (8). As one basic 20 physical property of solid material, hardness of three new AlAs polymorphs are calculated based on 21 Chen's empirical scheme in accordance with Eq. (9) [45].

24 The calculated $B, G, E, \sigma$, and $\kappa$ are all presented in Table 3. The value of $B$ agrees well with 25 the fitted value of derived from the BM-EOS, suggesting the calculation is correct. $B$ and $G$ 26 represents the capacity to resist volume change under load and reversible deformations upon shear 27 stress [46]. Among three newly discovered AlAs polymorphs, $o$ C12-AlAs exhibits the similar $B, G$ 28 and $E$ with $h$ P6-AlAs, whereas $c$ 24-AlAs has the smallest $B, G$ and $E$. Generally speaking, a higher 
$1 E$ denotes a stiffer material, which indicating that $h$ P6-AlAs and $o$ C12-AlAs are both stiffer than

2 cI24-AlAs. A similar conclusion is achieved in . Generally speaking, for solid material, larger 3 volume means smaller density and larger volume compressibility, imply smaller mechanical 4 modulus, and lower hardness, the relationship has also confirmed by oC12-, hP6-, and cI24-AlAs .

5 The calculated $B, G, E$ and signify that $o \mathrm{C} 12-, h \mathrm{P} 6-$, and $c \mathrm{I} 24-\mathrm{AlAs}$ possess appropriate

6 mechanical properties and these AlAs compound polymorphs extend the industrial applications of 7 aluminum compounds.

9 Table 3

10 BM-EOS fitting results (, , and ), shear anisotropic factors (, , and ), and mechanical parameters $(B$, $11 G, \kappa, E, \mu$, and ) for three new AlAs polymorphs. , $B, G, E$, and are expressed in GPa; whereas is 12 expressed in $\AA^{3}$, and the others hold dimensionless units.

\begin{tabular}{lllllllllllll}
\hline structure & & & & & & & $B$ & $G$ & $\kappa$ & $E$ & $\mu$ \\
\hline$o$ C12-A1As & 74.2 & 3.90 & 42.06 & 0.983 & 0.895 & 1.004 & 74.0 & 43.0 & 0.581 & 108.1 & 0.257 & 6.57 \\
$h$ P6-AlAs & 75.6 & 3.89 & 41.97 & 1.037 & - & 1.000 & 74.8 & 43.6 & 0.583 & 109.5 & 0.256 & 6.68 \\
$c \mathrm{I} 24-A 1 A s$ & 69.1 & 3.95 & 45.30 & 1.526 & - & - & 67.4 & 35.4 & 0.525 & 90.4 & 0.277 & 4.59 \\
\hline
\end{tabular}

14 3.5. Electronic properties

15 Based on the hybrid functional HSE06, the band structures of zb-, oC12-, hP6-, and $c$ I24-AlAs 16 at zero pressure are calculated and presented in Fig. 5. For zb-AlAs (Fig. 5a), the valence band 17 maximum $(\mathrm{VBM})$ and conduction band minimum $(\mathrm{CBM})$ are located at $\mathrm{G}$ point and $\mathrm{X}$ points, 18 respectively. The VBM and CBM are separated by a forbidden band of $2.081 \mathrm{eV}$, in agreement with 19 the earlier theoretical value (2.1 eV [47], $2.24 \mathrm{eV}$ [48]) and experimental value (2.16 eV [49], $2.2 \mathrm{eV}$ 20 [50] and $2.23 \mathrm{eV}$ [51]). At zero pressure, oC12-AlAs (Fig. 5b) and hP6-AlAs (Fig. 5c) are both 21 semiconductors with indirect band gaps of $0.468 \mathrm{eV}$ and $1.356 \mathrm{eV}$, respectively. For $o \mathrm{C} 12-\mathrm{AlAs}$, $22 \mathrm{VBM}$ and $\mathrm{CBM}$ are located at $\mathrm{Z}$ point and $\mathrm{Y}$ points, respectively. For $h \mathrm{P} 6-\mathrm{AlAs}$, VBM is located at 23 G point and CBM at M point, respectively. $c$ I24-AlAs (Fig. 5d) is also a semiconductor with a direct 24 band gap $(1.761 \mathrm{eV})$ at $\mathrm{G}$ point. 

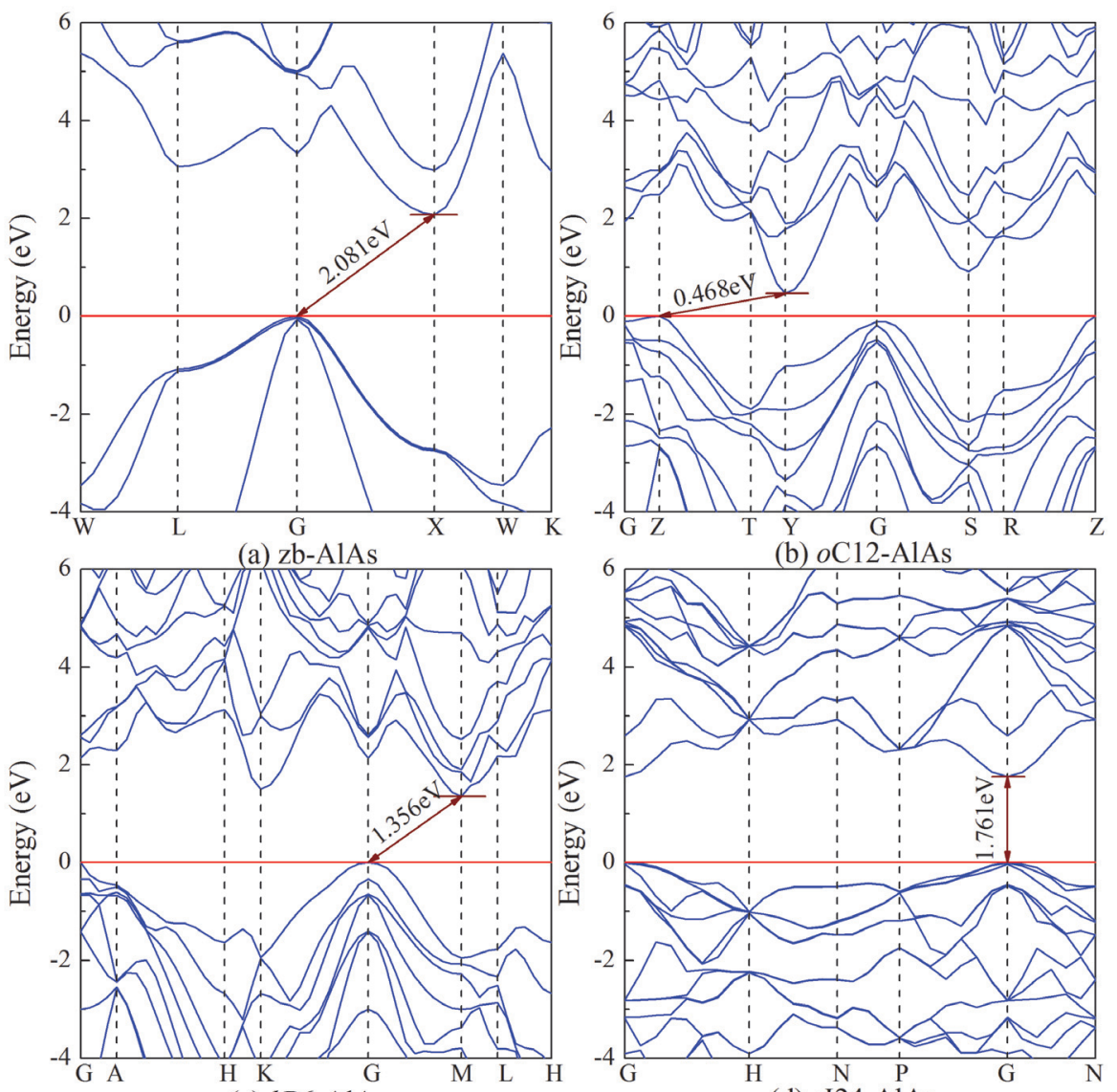

(c) $h \mathrm{P} 6-\mathrm{AlAs}$

(d) $c$ I24-AlAs

2 Fig. 5. Calculated band structures for zb-AlAs (a), oC12-AlAs (b), hP6-AlAs (c), and $c$ I24-AlAs (d)

3 at ambient pressure. The Fermi level is represented by a horizontal red line.

\section{Conclusion}

In summary, $o \mathrm{C} 12-, h \mathrm{P} 6-$, and $c \mathrm{I} 24-\mathrm{AlAs}$ are proposed by employing CALYPSO. The elastic constants and phonon dispersion spectra are calculated to certify the mechanical and dynamic stabilities of the three newly discovered phases. Based on calculated enthalpy curves relative to zb-AlAs as functions of pressure for various AlAs polymorphs, $o \mathrm{C} 12-, h \mathrm{P} 6-$, and $c \mathrm{I} 24-\mathrm{AlAs}$ all hold

10 energy advantages over those of cmcm-, and NiAs-AlAs at ambient pressure. The new AlAs

11 polymorphs are all pressure driven phases. zb-AlAs first transforms into NiAs-AlAs at 9.2 GPa and

12 then to cmcm-AlAs at $34.4 \mathrm{GPa}$. $o \mathrm{C12}-$, $h \mathrm{P} 6-$, and $c \mathrm{I} 24-\mathrm{AlAs}$ may be obtained by quenching

13 NiAs-AlAs with controlled unloading rate. Calculation for mechanical properties reveals that the

14 hardness of $o \mathrm{C} 12-$, and $h \mathrm{P} 6-\mathrm{AlAs}$ are similar, (6.57 GPa and 6.68 GPa, respectively), both higher

15 than that of $c \mathrm{I} 24-\mathrm{AlAs}(4.59 \mathrm{GPa})$. The shear anisotropic factors of $o \mathrm{C} 12-$, and $h \mathrm{P} 6-\mathrm{AlAs}$ are similar, 
1 and both smaller than that of $c$ I24-A1As. The electronic band structures calculation illustrate that all

2 the three newly discovered AlAs polymorphs are semiconductors with gaps smaller than that of 3 zb-AlAs. $o$ C12-AlAs and $h \mathrm{P} 6$-AlAs are both indirect semiconductors with band gaps $0.468 \mathrm{eV}$ and

$4 \quad 1.356 \mathrm{eV}$, respectively. $c \mathrm{I} 24-\mathrm{AlAs}$ is a direct semiconductor with a gap value of $1.761 \mathrm{eV}$.

5

6 Acknowledgements

This work was supported by the National Science Foundation of China (Grant Nos. 51421091 8 and 51525205), which is gratefully acknowledged.

\section{References}

11 [1] W. Martienssen, H. Warlimont, in, Springer, Heidelberg, 2005.

12 [2] S. Adachi, J. Appl. Phys., 58 (1985) R1-R29.

13 [3] L. Guo, J. Nanopart. Res., 13 (2011) 2029-2039.

14 [4] S. Froyen, M.L. Cohen, Phys. Rev. B, 28 (1983) 3258-3265.

15 [5] B. Weinstein, S. Hark, R. Burnham, R.M. Martin, Phys. Rev. Lett., 58 (1987) 781-784.

16 [6] U. Venkateswaran, L. Cui, B. Weinstein, F. Chambers, Phys. Rev. B, 45 (1992) 9237-9247.

17 [7] R.G. Greene, H. Luo, T. Li, A.L. Ruoff, Phys. Rev. Lett., 72 (1994) 2045-2048.

18 [8] A. Onodera, M. Mimasaka, I. Sakamoto, J. Okumura, K. Sakamoto, S. Uehara, K. Takemura, O.

19 Shimomura, T. Ohtani, Y. Fujii, J. Phys. Chem. Solids, 60 (1999) 167-179.

20 [9] G. Liu, Z. Lu, B.M. Klein, Phys. Rev. B, 51 (1995) 5678-5681.

21 [10] J. Cai, N.X. Chen, Phys. Rev. B, 75 (2007) 174116.

22 [11] A. Mujica, R.J. Needs, A. Munoz, Phys. Rev. B, 52 (1995) 8881-8892.

23 [12] A. Mujica, P. Rodríguez-Hernández, S. Radescu, R. Needs, A. Munoz, Phys. Status Solidi B, $24211(1999) 39-43$.

25 [13] S. Wang, H. Ye, J. Phys.: Condens. Matter, 14 (2002) 9579-9587.

26 [14] A. Srivastava, N. Tyagi, High Pressure Res., 32 (2012) 43-47.

27 [15] Y.C. Wang, J.A. Lv, L. Zhu, Y.M. Ma, Phys. Rev. B, 82 (2010) 094116. 
1 [16] Y.C. Wang, J. Lv, L. Zhu, Y.M. Ma, Comput. Phys. Commun., 183 (2012) 2063-2070.

2 [17] H. Wang, Y. Wang, J. Lv, Q. Li, L. Zhang, Y. Ma, Comput. Mater. Sci., 112, Part B (2016) 3 406-415.

4 [18] C. Liu, M. Hu, K. Luo, L. Cui, D. Yu, Z. Zhao, J. He, Comput. Mater. Sci., 117 (2016) 496-501.

5 [19] L. Zhu, H. Liu, C.J. Pickard, G. Zou, Y. Ma, Nat. Chem., 6 (2014) 644-648.

6 [20] J.H. Wu, G. Yang, Comput. Mater. Sci., 82 (2014) 86-91.

7 [21] P. Sarker, M.N. Huda, Comput. Mater. Sci., 111 (2016) 359-365.

[22] C. Liu, Z. Zhao, K. Luo, M. Hu, M. Ma, J. He, Diamond Relat. Mater., (2016).

[23] S.J. Clark, M.D. Segall, C.J. Pickard, P.J. Hasnip, M.J. Probert, K. Refson, M.C. Payne, Z. Kristallogr., 220 (2005) 567-570.

11 [24] W. Kohn, L.J. Sham, Phys. Rev., 140 (1965) A1133-A1138.

12 [25] J.P. Perdew, K. Burke, M. Ernzerhof, Phys. Rev. Lett., 77 (1996) 3865-3868.

13 [26] D. Vanderbilt, Phys. Rev. B, 41 (1990) 7892-7895.

14 [27] H.J. Monkhorst, J.D. Pack, Phys. Rev. B, 13 (1976) 5188-5192.

15 [28] A.J. Garza, G.E. Scuseria, J. Phys. Chem. Lett., 7 (2016) 4165-4170.

16 [29] A.V. Krukau, O.A. Vydrov, A.F. Izmaylov, G.E. Scuseria, J. Chem. Phys., 125 (2006) 224106.

17 [30] S. Baroni, S. de Gironcoli, A. Dal Corso, P. Giannozzi, Rev. Mod. Phys., 73 (2001) 515-562.

18 [31] Z. Wu, E. Zhao, H. Xiang, X. Hao, X. Liu, J. Meng, Phys. Rev. B, 76 (2007) 054115.

[32] H.Y. Wang, X.S. Li, C.Y. Li, K.F. Wang, Mater. Chem. Phys., 117 (2009) 373-376.

[33] A. Srivastava, N. Tyagi, U.S. Sharma, R.K. Singh, Mater. Chem. Phys., 125 (2011) 66-71.

[34] B. Amrani, Superlattices Microstruct., 40 (2006) 65-76.

[35] J. Crain, G. Ackland, J. Maclean, R. Piltz, P. Hatton, G. Pawley, Phys. Rev. B, 50 (1994) $13043-13046$

[36] C.S. Menoni, J.Z. Hu, I.L. Spain, Phys. Rev. B, 34 (1986) 362-368.

[37] Y. Zhao, F. Buehler, J.R. Sites, I.L. Spain, Solid State Commun., 59 (1986) 679-682.

[38] R. Nelmes, M. McMahon, N. Wright, D. Allan, J. Loveday, Phys. Rev. B, 48 (1993) 9883-9886.

[39] F. Birch, J. Appl. Phys., 9 (1938) 279-288.

[40] S.I. Ranganathan, M. Ostoja-Starzewski, Phys. Rev. Lett., 101 (2008) 055504. 
1 [41] C. Liu, M. Hu, K. Luo, D. Yu, Z. Zhao, J. He, J. Appl. Phys., 119 (2016) 185101.

2 [42] J.P. Watt, J. Appl. Phys., 50 (1979) 6290-6295.

3 [43] J.P. Watt, L. Peselnick, J. Appl. Phys., 51 (1980) 1525-1531.

4 [44] Z. Wu, E. Zhao, H. Xiang, X. Hao, X. Liu, J. Meng, Phys. Rev. B, 76 (2007) 054115.

5 [45] X.Q. Chen, H.Y. Niu, D.Z. Li, Y.Y. Li, Intermetallics, 19 (2011) 1275-1281.

6 [46] H.B. Ozisik, K. Colakoglu, E. Deligoz, H. Ozisik, J. Mol. Model., 18 (2012) 3101-3012.

7 [47] T. Shimazaki, Y. Asai, J. Chem. Phys., 132 (2010) 224105.

8 [48] J. Heyd, J.E. Peralta, G.E. Scuseria, R.L. Martin, J. Chem. Phys., 123 (2005) 113-113.

9 [49] S.M. Sze, K.K. Ng, Physics of semiconductor devices, John wiley \& sons, 2006.

10 [50] H. Minden, Appl. Phys. Lett., 17 (1970) 358-360.

11 [51] P.Y. Yu, M. Cardona, L.J. Sham, Semiconductor Science \& Technology, 50 (2011) 76. 